\title{
SIGMA-AMENABLE LOCALLY COMPACT GROUPS ${ }^{1}$
}

\author{
J. W. JENKINS
}

ABSTRACT. Let $G$ denote a locally compact group and $\sigma(G)$ the semigroup of nonempty compact subsets of $G$. The combinatorial properties of the family of all groups $G$ for which $\sigma(G)$ is amenable is studied. The relationship between amenability of $G$ and amenability of $\sigma(G)$ is also investigated.

1. $\sigma$-amenability. Let $S$ be a semigroup and $X$ a closed subspace of $m(s)$, the space of all bounded real valued functions defined of $S$ with the sup norm. Assume that $X$ contains the constant functions. A linear functional $\mu$ on $X$ is a mean if $\mu$ is positive and of norm one. If for each $f$ in $X$, ${ }_{s} f$ is also in $X$, where ${ }_{s} f(t)=f(s t)$ for each $s, t$ in $S$, and if $\mu\left({ }_{s} f\right)=\mu(f)$ for each $f$ in $X$ and $s$ in $S$, then $\mu$ is a left invariant mean (LIM) on $X$.

If $G$ is a locally compact group, $G$ is said to be amenable (more accurately, left-amenable) if there is a LIM on the space of bounded continuous functions.

For $G$ a locally compact group, we denote by $\sigma(G)$ the family of all nonempty compact subsets of $G . \sigma(G)$ becomes a semigroup if we define multiplication by set product, i.e. $U U^{\prime}=\left\{u u^{\prime} \mid u \in U, u^{\prime} \in U^{\prime}\right\}$ for each $U, U^{\prime}$ in $\sigma(G)$.

Definition 1.1 . A locally compact group $G$ is said to be $\sigma$-amenable if there is a LIM on $m(\sigma(G))$.

For the remainder of this section we are primarily concerned with the combinatorial properties of $\sigma$-amenable groups. $G$ and $H$ will denote locally compact Hausdorff groups. We begin with

Proposition 1.2. If $G$ is an abelian group or a compact group, then $G$ is $\sigma$-amenable.

Proof. If $G$ is an abelian group then quite obviously $\sigma(G)$ is commutative, and hence there is a LIM on $m(\sigma(G))$ (see Day [1]).

Suppose that $G$ is compact. Then $G \in \sigma(G)$ and hence the point evaluation $e_{G}$, defined by $e_{G}(f)=f(G)$ for each $f$ in $m(\sigma(G))$ is a mean

Received by the editors June 15, 1970.

AMS 1970 subject classifications. Primary 22-XX, 22D05; Secondary 20M05, $20 \mathrm{M} 99$.

Key words and phrases. Amenable semigroups, semigroup of compact subsets, $\sigma$ amenable groups, free subsemigroups, free product, disjoint right ideals.

1 This research was partially supported by National Science Foundation Research Grant 12027 and GU-3171. 
on $m(\sigma(G))$. Furthermore, since for each $U$ in $\sigma(G)$ we have $U G$ $=G, e_{G}(v f)=f(U G)=f(G)=e_{G}(f)$. Thus, $e_{G}$ is a LIM on $m(\sigma(G))$.

Proposition 1.3. Let $\pi$ be an open continuous homomorphism of $G$ onto $H$, and suppose that $G$ is $\sigma$-amenable. Then $H$ is $\sigma$-amenable.

Proof. $\pi$ has an obvious extension to a homomorphism $\bar{\pi}$ of $\sigma(G)$ into $\sigma(H)$. The fact that $\bar{\pi}$ is an epimorphism is an immediate consequence of $\pi$ being open and continuous. The proposition then follows from Day [1].

Proposition 1.4. If $H$ is an open subgroup of $G$ and if $G$ is $\sigma$-amenable then $H$ is $\sigma$-amenable.

Proof. First observe that if $U \in \sigma(G)$, then there exist $g_{1}, g_{2}$, $\cdots, g_{n}$ in $G$ such that $U \subset \cup_{i=1}^{n} H g_{i}$. For each $f$ in $m(\sigma(H))$, define $\bar{f}$ in $m(\sigma(G))$ pointwise by

$$
\bar{f}(U)=\frac{1}{n} \sum_{i=1}^{n} f\left(U g_{i}^{-1} \cap H\right),
$$

where $U \cap H g \neq \varnothing \Leftrightarrow H g=H g_{i}$ for some $1 \leqq i \leqq n$, and $H g_{i}=H g_{j} \Leftrightarrow i=j$. Let $\mu$ be a LIM on $m(\sigma(G))$ and define $\bar{\mu}$ in $m^{*}(\sigma(H))$ by $\bar{\mu}(f)=\mu(\bar{f})$ for each $f$ in $m(\sigma(H))$. It is easily seen that $\bar{\mu}$ is a mean on $m(\sigma(H))$. Suppose now that $V \in \sigma(H)$ and that $f \in m(\sigma(H))$. Then $\bar{\mu}\left({ }_{V} f\right)=\mu(v \bar{f})$, and, for each $U \in \sigma(G)$,

$$
\begin{aligned}
\nabla \bar{f}(U) & =\frac{1}{n} \sum_{i=1}^{n} \nabla f\left(U g_{i}^{-1} \cap H\right)=\frac{1}{n} \sum_{i=1}^{n} f\left[V\left(U g_{i}^{-1} \cap H\right)\right] \\
& =\frac{1}{n} \sum_{i=1}^{n} f\left(V U g_{i}^{-1} \cap H\right)={ }_{V}(\bar{f})(U) .
\end{aligned}
$$

Hence, $\bar{\mu}(v f)=\mu(v(\bar{f}))=\mu(\bar{f})=\bar{\mu}(f)$, and $\bar{\mu}$ is a LIM on $m(\sigma(H))$.

Proposition 1.5. If $G$ is a directed union of open $\sigma$-amenable subgroups $\left\{H_{\alpha}\right\}$ then $G$ is $\sigma$-amenable.

PRoof. If $\mu_{\alpha}$ is a LIM on $m\left(\sigma\left(H_{\alpha}\right)\right)$ then $\bar{\mu}_{\alpha}(f)=\mu_{\alpha}(f \mid \sigma(H))$ is a mean on $m(\sigma(G))$ that is invariant under translations by elements of $\sigma\left(H_{\alpha}\right)$. Since $\left\{H_{\alpha}\right\}$ is directed, the family $\left\{M_{\alpha}\right\}$, where $M_{\alpha}$ is the compact subset of means on $m(\sigma(G))$ that are $\sigma\left(H_{\alpha}\right)$ invariant, has the finite intersection property. Thus, since the means on $m(\sigma(G))$ is a compact set, $\cap M_{\alpha} \neq \varnothing$. Finally, since each $H_{\alpha}$ is open in $G$, and $G=\cup H_{\alpha}$, each $U$ in $\sigma(G)$ is in $\sigma\left(H_{\alpha}\right)$ for some $\alpha$. Thus, if $\mu \in \cap M_{\alpha}$, $\mu$ is a LIM on $m(\sigma(G))$. 
The preceding propositions indicate that the family of $\sigma$-amenable groups has combinatorial properties similar to the family of amenable groups. However, there is one significant difference. The family of amenable groups is closed with respect to group extensions. This is not true for $\sigma$-amenable groups, as an example in $\$ 2$ will show. ${ }^{2}$ In the following proposition it is shown that certain group extensions do give rise to $\sigma$-amenable groups.

Proposition 1.6. Suppose that $H$ is the directed union of open, compact, normal subgroups of $G$ and that $G / H$ is $\sigma$-amenable. Then $G$ is $\sigma$-amenable.

Proof. First note that $H$ is an open normal subgroup of $G$, and hence $G / H$ is a discrete group.

Let $\left\{g_{\alpha}\right\}$ be a transversal of $G / H$, and let $H=\cup_{\beta \in B} H_{\beta}$, where each $H_{\beta}$ is an open compact normal subgroup of $G$.

For each $\beta$ in $B$ and $f$ in $m(\sigma(G))$, define $f_{\beta}$ in $m(\sigma(G))$ by $f_{\beta}(U)$ $=f\left(U H_{\beta}\right)$. Define $\bar{f}_{\beta}$ in $m(\sigma(G / H))$ by

$$
\bar{f}_{\beta}\left(\left\{g_{\alpha_{1}} H, \cdots, g_{\alpha_{n}} H\right\}\right)=f_{\beta}\left(\left\{g_{\alpha_{1}}, \cdots, g_{\alpha_{n}}\right\}\right) .
$$

Let $\lambda$ be a LIM on $m(\sigma(G / H))$, and define the mean $\mu_{\beta}$ on $m(\sigma(G))$ by $\mu_{\beta}(f)=\lambda\left(\bar{f}_{\beta}\right)$.

Let $U \in \sigma(G)$. There exist $g_{\alpha_{i}} \in\left\{G_{\alpha}\right\}$ and subsets $K_{i}$ of $H$, for $i=1, \cdots, n$, such that

$$
U=\bigcup_{i=1}^{n} g_{\alpha_{i}} K_{i}
$$

Now,

$$
\mu_{\beta}(U f)=\lambda\left(\overline{(U f)_{\beta}}\right),
$$

and

$$
\begin{array}{r}
\overline{(v f)_{\beta}}\left(\left\{g_{\alpha_{1}^{\prime}} H, \cdots, g_{\alpha_{m}^{\prime}} H\right\}\right)=\left({ }_{U} f\right)_{\beta}\left(\left\{g_{\alpha_{1}^{\prime}}, \cdots, g_{\alpha_{m}^{\prime}}\right\}\right)=U f\left(\bigcup_{i=1}^{m} g_{\alpha_{i}^{\prime}} H_{\beta}\right) \\
=f\left(\bigcup_{i=1}^{m} U H_{\beta} g_{\alpha_{i}^{\prime}}\right)=f\left[\bigcup_{i=1}^{m}\left(\bigcup_{j=1}^{n} g_{\alpha_{j}} K_{j}\right) H_{\beta} g_{\alpha_{i}^{\prime}}\right] .
\end{array}
$$

for $\beta$ sufficiently large, $K_{j} \subset H_{\beta}$ for $j=1,2, \cdots, m$, and

$$
\overline{(v f)_{\beta}}\left(\left\{g_{\alpha_{1}^{\prime}} H, \cdots, g_{\alpha_{m}^{\prime}} H\right\}\right)=f\left(\bigcup_{i=1}^{m}\left(\bigcup_{j=1}^{n} g_{\alpha_{j}} g_{\alpha_{i}^{\prime}} H_{\beta}\right)\right) \text {. }
$$

2 The author wishes to thank E. Granirer for pointing out an error in a proof of this proposition. 
If $U \in \sigma(G)$ and $U=\bigcup_{i=1}^{n} g_{\alpha_{i}} K_{i}$, define $\tilde{U}$ in $\sigma(G / H)$ by $\tilde{U}$ $=\left\{g_{\alpha_{1}} H, \cdots, g_{\alpha_{n}} H\right\}$. Then,

$$
\begin{aligned}
& \tilde{U}\left(\bar{f}_{\beta}\right)\left(\left\{g_{\alpha_{1}^{\prime}} H, \cdots, g_{\alpha_{m}^{\prime}} H\right\}\right)=\bar{f}_{\beta}\left(\tilde{U}\left\{g_{\alpha_{1}^{\prime}} H, \cdots, g_{\alpha_{m}^{\prime}} H\right\}\right) \\
& \quad=f_{\beta}\left(\left\{g_{\alpha_{1}}, \cdots, g_{\alpha_{n}}\right\}\left\{g_{\alpha_{1}^{\prime}}, \cdots, g_{\alpha_{n}^{\prime}}\right\}\right)=f\left(\bigcup_{i=1}^{n}\left(\bigcup_{j=1}^{m} g_{\alpha_{i}} g_{\alpha_{j}^{\prime}} H_{\beta}\right)\right) .
\end{aligned}
$$

Therefore, given $U$ in $\sigma(G)$, for sufficiently large $\beta$ we have

$$
\left.\mu_{\beta}(\tilde{U} f)=\lambda(\overline{(U f})_{\beta}\right)=\lambda\left(\tilde{U}\left(\bar{f}_{\beta}\right)\right)=\lambda\left(\bar{f}_{\beta}\right)=\mu_{\beta}(f) .
$$

Any $w^{*}$-cluster point of $\left\{\mu_{\beta}\right\}$ is thus a LIM on $m(\sigma(G))$.

2. Amenability and $\sigma$-amenability. In this section we consider the relationships of amenable and $\sigma$-amenable groups.

Given a group $G$, let $\sigma_{0}(G)$ denote the set of all elements of $\sigma(G)$ which have nonempty interiors. We have

LEMMA 2.1. There is a LIM on $\sigma(G)$ if, and only if, there is a LIM on $\sigma_{0}(G)$.

Proof. Merely observe that $\sigma_{0}(G)$ is an ideal of $\sigma(G)$. The lemma then follows from Wilde and Witz [6].

Proposition 2.2. If $G$ is $\sigma$-amenable then $G$ is amenable.

Proof. For each $f$ in $C B(G)$, the bounded continuous function on $G$, define $\bar{f}$ in $m\left(\sigma_{0}(G)\right)$ by

$$
\bar{f}(U)=|U|^{-1} \int_{U} f(t) d t
$$

for each $U$ in $\sigma_{0}(G)$, where $d t$ denotes left Haar measure on $G$ and $|U|$ denotes the measure of $U$.

By Lemma 2.1, there is a LIM $\mu$ on $m\left(\sigma_{0}(G)\right)$. Define $\bar{\mu}$ in $C B(G)^{*}$ by $\bar{\mu}(f)=\mu(\bar{f})$ for each $f$ in $C B(G)$. It is obvious that $\bar{\mu}$ is a mean. If $g \in G$,

$$
\bar{\mu}\left({ }_{\sigma} f\right)=\mu\left({ }_{\sigma} \bar{f}\right),
$$

and

$$
\bar{\sigma} \bar{f}(U)=|U|^{-1} \int_{U} f(g t) d t=|U|^{-1} \int_{o U} f(t) d t={ }_{\sigma}(\bar{f})(U)
$$

for each $U$ in $\sigma_{0}(G)$. Hence

$$
\bar{\mu}\left({ }_{\ell} f\right)=\mu\left({ }_{0}(\bar{f})\right)=\mu(\bar{f})=\bar{\mu}(f)
$$

for each $f$ in $C B(G)$, and thus $G$ is amenable. 
Proposition 2.3. Let $G=Z_{2} * Z_{2}$, the free product of $Z_{2}$ with $Z_{2}$. Then, $G$ is not $\sigma$-amenable.

Proof. $G$ is isomorphic to the discrete group generated by $a$ and $b$ where $a^{2}=b^{2}=e$. We show that $G$ is not $\sigma$-amenable by producing disjoint right ideals in $\sigma(G)$.

Let $U=\{a, a b\}$ and $V=\{b, b a\}$. Then $U \sigma(G) \cap V \sigma(G)=\varnothing$. To see this consider the following: each element $g$ in $G$ can be written in

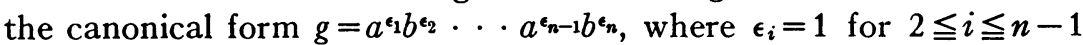
and $\left\{\epsilon_{1}, \epsilon_{n}\right\} \subset\{0,1\}$. Define $\rho(g)=\sum_{i=1}^{n} \epsilon_{i}$, and for each $U$ in $\sigma(G)$, define $\rho(U)=\max \{\rho(g) \mid g \in U\}$.

If $W \in U \sigma(G)$, and $g \in W$ such that $\rho(g)=\rho(W)$ then one can easily see that in the canonical form of $g, \epsilon_{1}=1$. If $W \in V \sigma(G)$, and $g$ is chosen similarly, $\epsilon_{1}=0$ in the canonical form of $g$. Thus $U \sigma(G) \cap V \sigma(G)=\varnothing$. Since $U \sigma(G)$ and $V \sigma(G)$ are right ideals of $\sigma(G)$, the proposition follows.

REMARK. It is well known that $Z_{2} * Z_{2}$ is amenable (cf. Dixmier [2]). We can therefore conclude from the preceding propositions that the family of $\sigma$-amenable groups is properly contained in the family of amenable groups.

Proposition 2.3 also shows that some restrictions on the subgroup $H$ of Proposition 1.6 are necessary: $Z_{2} * Z_{2}$ contains a normal abelian subgroup, generated by $a b$, of finite index. Hence, not every extension of a $\sigma$-amenable group by a $\sigma$-amenable group is $\sigma$-amenable.

There are amenable groups which have nonamenable open subsemigroups (cf. Hochster [4], Jenkins [5]). Let $A^{\prime}$ be the family of all (amenable) groups such that each open subsemigroup is amenable, and let $A$ denote the family of all $\sigma$-amenable groups. It is natural to ask if there is a inclusion relation between $A$ and $A^{\prime}$. Although the answer is not known, we have the following information.

Proposition 2.4. $A^{\prime}$ is not contained in $A$.

Proof. $Z_{2} * Z_{2}$ is an example of an element of $A^{\prime}$ that is not in $A$. To prove that $Z_{2} * Z_{2}$ is in $A^{\prime}$ it suffices to show that $Z_{2} * Z_{2}$ contains no free subsemigroups on two generators (cf. Frey [2]). Assume the contrary, and let $g$ and $h$ be elements of $Z_{2} * Z_{2}$ that generate a free subsemigroup. We may assume that in canonical form $g=a^{\epsilon_{1}} b^{\epsilon_{2}}$. . $a^{\epsilon_{n-1}} b^{\epsilon_{n}}$ where $\epsilon_{i}=1$ for $1 \leqq i \leqq n-1$. Note that $\epsilon_{n}=1$ also, for if not, $g^{2}=e$. If in canonical form $h=a^{\epsilon_{1}} b^{\epsilon_{2}} \cdots a^{\epsilon_{m-1}} b_{m}^{\epsilon_{m}}$ where $\epsilon_{i}=1$ for $1 \leqq i \leqq m$, then $g^{m}=h^{n}$, a contradiction. Thus, we assume that $h$ $=b^{\epsilon_{1}} a^{\epsilon_{2}} \cdots b^{\epsilon_{m-1}} a^{\epsilon_{m}}$ where $\epsilon_{i}=1$ for $1 \leqq i \leqq m$. Then (i) if $n>m$, $h g=a^{\epsilon_{1}} b^{\epsilon_{2}} \cdots b^{\epsilon_{n-m}}$ where $\epsilon_{i}=1$ for $1 \leqq i \leqq n-m$, (ii) if $m>n$, $h g$ 
$=b^{\epsilon_{1}} \cdots a^{\epsilon_{m-n}}$ where $\epsilon_{i}=1$ for $1 \leqq i \leqq m-n$, or (iii) $h g=e$. If (i) holds then $(h g)^{(n}=g^{n-m}$, a contradiction. If (ii) holds, $(h g)^{m}=h^{m-n}$, a contradiction. Finally, if (iii) holds, then $h g=g h$, again a contradiction. Therefore the semigroup generated by $g$ and $h$ is not free, and $Z_{2} * Z_{2} \in A^{\prime}$.

\section{REFERENCES}

1. M. M. Day, Amenable semigroups, Illinois J. Math. 1 (1957), 509-544. MR $19,1067$.

2. J. Dixmier, Les moyennes invariantes dans les semi-groupes et leurs applications, Acta. Sci. Math. (Szeged) 12 (1950), pars A, 213-227. MR 12, 267.

3. A. H. Frey, Studies in amenable semigroups, Thesis, University of Washington, Seattle, Washington, 1960.

4. M. Hochster, Subsemigroups of amenable groups, Proc. Amer. Math. Soc. 21 (1969), 363-364. MR 39 \#1575.

5. J. W. Jenkins, Subsemigroups of an amenable group, Proc. Amer. Math. Soc. 25 (1970), 766-770.

6. C. Wilde and K. Witz, Invariant means and the Stone-Čech compactification, Pacific J. Math. 21 (1967), 577-586. MR 35 \#3423.

State University of New York at Albany, Albany, New York 12203 